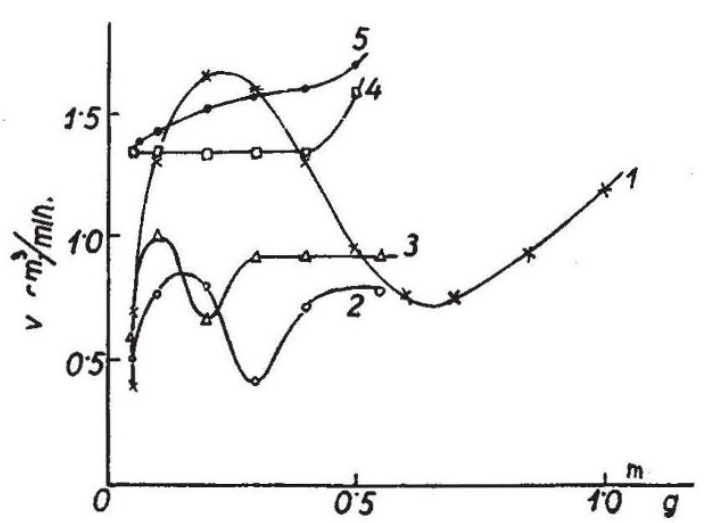

Rate of hydrogenation of a $0.13 \mathrm{~mol}$. sodium cinnamate solution (in $n \mathrm{~cm}^{3}$ consumed hydrogen per minute). Curve 1 , sodium cinnamate alone $(r=2.4)$; curve 2 , with an addition of $0.033 \mathrm{~mol}$ ammonium chloride $(r=3 \cdot 4)$; curve 3 , the same $(r=2 \cdot 7)$ curve 4 , the same $(r=1.6) ;$ curve 5 , the same $(r=1.0)$

If, on the other hand, the solution is vigorously shaken, the rate of the hydrogenation increases proportionally with the mass of the catalyst.

The shape of the curve $v=f(m)$ is different in the presence of ammonium salts in the solution, showing, in appropriate circumstances, neither maximum nor minimum, even if the stirring is not rapid. The shape of the curve is greatly influenced by $r$. Varying $r, v$ can be made independent of $m$; moreover, the maximum can also re-appear (see graph). The ions $\mathrm{Na}^{+}, \mathrm{H}^{+}$and $\mathrm{Cl}^{-}$have no such influence. It should be mentioned that the electrokinetic potential of the catalyst is essentially influenced by ammonium ions, suggesting that the alteration of the $v=f(m)$ curve is-partly at least-caused by this factor. The experimental results suggest the following explanation.

The fraction of the catalyst on the surface of the solution and near it has a greater catalytic action than the fraction in the solution. This behaviour can be correlated with various factors, one of which is the fact that the catalyst near the surface of the solution, which is in almost direct contact with the hydrogen gas, contains more adsorbed hydrogen than that in the solution, the rate of the hydrogenation being, ceteris paribus, undoubtedly proportional to the concentration of hydrogen per unit area of the surface of catalyst. Within the solution, the concentration of hydrogen is under stationary conditions lower than in equilibrium, having regard to the point that the reaction is consuming a certain amount of hydrogen, the support of which by diffusion and convection is rather slow.

The increase of mass of the catalyst has various effects on the rate of the reaction. On one hand, the quantity of the catalyst near the surface of the solution will be increased, accelerating the reaction, although the velocity of stirring, the pressure of hydrogen and surface area of solution are kept constant. The increased rate of hydrngenation near the surface diminishes, on the other hand, the diffusion of the hydrogen in the solution, since a greater part of the hydrogen dissolved per second from the gas will be consumed near the surface than in such experiments in which we are applying a smaller amount of catalyst, and only a smaller part of the hydrogen can be transferred into the solution. The concentration of the hydrogen is consequently diminished in the solution, and the adsorbed quantity per unit area of the catalyst diminishes also, all the more so as the whole area of the catalyst is increased.

The effect in the solution is twofold : on one hand, the increase of the whole area of the catalyst is accelerating the reaction; on the other, the decrease of the adsorbed amount of hydrogen per unit area of the catalyst is retarding it. It depends on the experimental conditions which of these two effects is to prevail. The rate of the reaction in the solution can therefore be diminished or increased by increasing the quantity of the catalyst. A more detailed development of this consideration shows that the shape of the theoretical curve $v=f(m)$ is, under adequate conditions, similar to that of the experimental curves. Further investigations on the quantitative agreement are in progress.

Fuller details of the above-mentioned experiments and considerations, carried out in collaboration with J. Szabo, will be published elsewhere.

Institute for Physical Chemistry, T. ERDEY-Grớz

Peter Pázmány University, Budapest. July 1948.

' Kailan, A., and Hartel, F., Monatshefte, 70, 329 (1937).

' Csürös, Z., Zech, K., and Géczy, I., Hungarica Acta Chimica, 1, I (1946). Cssürös,'Z., and Pfliegel, T., ibid., 1, 24 (1946).

\section{Sun-bathing by Birds and its Anti-rachitic Value}

IN the issue of Nature of November 27, p. 843, there is a note on the subject of sun-bathing by birds, quoting British Birds as saying that the question has been raised "whether irradiation of the skin with its consequent effect of vitamin $\mathrm{D}$ production would take place through sun-bathing".

In this connexion I should like to direct attention to the publications by $\mathrm{Hou}^{1,2}$ in 1930 on this subject. Hou found by feeding tests that the skin and feathers of fowls exposed to the sun in the usual way were antirachitic, but that if the birds' preen glands were removed, and vitamin D excluded from their diet, they developed rickets.

From these results we can reasonably assume that one of the functions of preening is to enable the bird to secure and swallow irradiated preen gland oil, which suggests that direct irradiation of the skin is not necessary for birds to keep them free from rickets.

It seems highly probable that, in the case of mammals which have a thick enough enat of hair or fur to prevent direct access of sunlight to the skin, the sebaceous secretion which lubricates the hair contains antecedents which are converted into vitamin $\mathbf{D}$ by irradiation. The elaborate toilet of the fur by such animals would therefure be com. parable physiologically with preening by birds. According to Hou and $\mathrm{Tso}^{3}$, human sebum is antirachitic, and Hou found that if rabbits' ears were washed with ether, irradiation of the ears by a mercury vapour lamp could not cure rickets induced in the animal, but that it could if the fatty substances were not removed.

\section{London Hospital Medical College, \\ Turner Street, \\ London, E.1. \\ Dec. 10.}

G. H. Bourne

1 Hou, H. C., Chin. J. Physiol., 4, 79 (1930).

${ }^{2}$ Hou, H. C., Chin. J. Physiol., 1, 345 (1930).

3 Hou, H. C., and Tso, E., Chin. J. Physiol., 4, 93 (1930). 